\title{
Heart rate dynamics and lactate following high- intensity race-pace continuous vs interval workouts in highly trained athletes
}

\author{
I. MARTINEZ-NAVARRO ${ }^{1,2 *}$ (D) A. MONTOYA ${ }^{1,2}$, M. MATEO-MARCH $^{3}$ and \\ C. BLASCO-LAFARGA ${ }^{1}$
}

${ }^{1}$ Physical Education and Sport Department, Faculty of Physical Activity and Sport Sciences, University of Valencia, Valencia, Spain

${ }^{2}$ Sports Health Unit, 9 de Octubre Vithas Hospital, Valencia, Spain

${ }^{3}$ University Miguel Hernandez, Elche, Spain

Received: September 28, 2020 • Accepted: December 21, 2020

Published online: June 28, 2021

(C) 2020 Akadémiai Kiadó, Budapest

\begin{abstract}
Purpose: The present study aimed to compare the physiological responses of high-intensity race-pace continuous vs. interval workouts commonly used in middle-distance athletics, by means of analyzing postexercise cardiac autonomic regulation and lactate. Methods: Nineteen highly-trained 800-m male runners were asked to run a $600-\mathrm{m}$ race-pace continuous workout and a $2 \times 4 \times 200$-m interval training, counterbalanced and randomized within one week of difference. Blood lactate jointly with linear and nonlinear heart rate dynamics were assessed during the immediate 15-min recovery. Age-category (Under23-Senior vs. Juvenile-Junior) was considered as an inter-subject factor. Results: Peak lactate was higher following the interval training $\left(15.51 \pm 0.99\right.$ vs $\left.13.83 \pm 1.77 \mathrm{mmol} \mathrm{L}^{-1} ; P<0.05\right)$ whereas lactate removal was almost nonexistent $15 \mathrm{~min}$ after both workouts (between 0 and 16\%). Vagal modulation (ln RMSSD and lnRMSSD to RR ratio) remained significantly depressed at the end of recovery following both workouts, although the alteration was larger following the interval training. Detrended Fluctuation Analysis evidenced a more random HR behavior ( $\mathrm{DFA}_{1}$ closer to 0.5 ) during the first 9 min of recovery after the interval training, whereas no significant change was observed in heart rate complexity (SampEn). Neither were differences found in post-exercise lactate and HR dynamics as a function of age-category. Conclusions: High-intensity workouts commonly used in middle-distance athletics, both race-pace continuous and
\end{abstract}

${ }^{*}$ Corresponding author. Department of Physical Education and Sports, Faculty of Physical Activity and Sport Sciences, University of Valencia, C/Gascó Oliag, 3, 46010, Valencia, Spain. Tel.: +34 667331613. E-mail: Ignacio. Martinez-Navarro@uv.es 
intervallic approaches, induce a large depression of vagal modulation in highly trained runners, although interval trainings appear to induce even a greater alteration of both linear and nonlinear HR dynamics and a higher post-exercise peak lactate.

\section{KEYWORDS}

complexity, middle-distance athletics, recovery, supramaximal, running

\section{INTRODUCTION}

In the acute period leading up to the major races of the season, highly-trained middle-distance athletes (i.e., 800 and 1500-m runners) usually alternate race-pace continuous and interval workouts performed at intensities very close or above their competitive pace to effectively prepare for the competition. As a rule $[1,2]$, race-pace continuous workouts, inasmuch as they are intended to resemble the whole nature of the event and tackling the athlete's pacing ability, consist of a single repetition conducted at competitive pace on a distance close to the real competition (usually between 50 and 75\%). Meanwhile, interval approaches are employed to improve speed-endurance and enhance the athlete's ability to recover between repetitions. They consist of sets of shorter runs performed at intensities above the athlete's competitive pace. The total distance covered in this latter workout typically varies from 2 to 3 times that of the event [3, 4]. Both approaches meet the idea that high-intensity training is still able to enhance traininginduced adaptations, while high training volumes are reduced before the major competitions [5]. However, it is difficult to speculate as to which of the two approaches stresses the cardiac autonomic regulation and the lactate production energy system to a greater extent. The response to this issue is of particular importance, both to quantify the suitability of each workout and to measure the training load associated with each one, insofar as cardiac autonomic recovery has been stated to reflect restoration of cardiovascular homeostasis [6].

In this regard, several investigations have analyzed immediate post-exercise Heart Rate Variability (HRV) following interval vs. continuous approaches [7-12]. The majority of studies showed that HRV was disturbed to a greater extent following interval exercises $[7,8,11,12]$, although Cyprian and Vala [9] failed to find significant differences in post-exercise HRV between interval and continuous workouts, whereas Kaikkonen et al. [10]. concluded that when interval and continuous interventions were performed at a similar workload, HRV was lower after continuous training. However, none of these studies employed continuous running workouts performed at velocities above that associated with $\dot{\mathrm{V}} \mathrm{O}_{2} \max \left(\mathrm{vV}_{2 \max }\right)$; whereas race-pace continuous workouts used in $800-\mathrm{m}$ training do meet this requirement, since 800 -m average racing velocity is above $\mathrm{v}_{\mathrm{V}_{2}} \max$ [13]. Moreover, it appears that intermittent workouts provoke larger lactate concentrations. Saraslanidis et al. [2] analyzed running workouts usually employed in 400-m training, and reported that post-exercise lactate was higher after high-intensity interval workouts compared with continuous ones of the same total distance. Similarly, Buchheit et al. [7, 8] showed that blood lactate was significantly higher after interval protocols in comparison with continuous ones. Notwithstanding, in these three studies lactate was measured only once following the exercise, so it seems of interest to assess lactate concentrations during a longer 
recovery period, jointly with post-exercise heart rate (HR) dynamics. Moreover, the assessment of nonlinear oscillations (i.e. complexity and fractal properties) offers an additional qualitative insight into HR dynamics, characterized by less dependency on the HR itself and lower interindividual and intraindividual variation, as compared with traditional time domain (i.e., the standard deviation and the root-mean-square difference of successive normal RR intervals) and spectral analysis measures (i.e., high and low frequency power bands) [14]. Indeed, changes in complexity and fractal properties have been previously reported in the absence of changes in linear indices, suggesting that nonlinear methodologies may also be more sensitive [15].

Therefore, the main purpose of the present study was to evaluate (a) HR dynamics during the immediate 15-min post-exercise and (b) peak lactate and 15-min post-exercise lactate clearance following two different running workouts usually employed in 800-m training: a 600-m race-pace continuous workout $(\mathrm{CON})$ and a $2 \times 4 \times 200$-m speed-endurance interval training (INT), to ascertain whether this time lapse is enough to enable HR dynamics and lactate clearance, and to determine which of these two approaches stresses the cardiac autonomic regulation and the lactate production energy system to a greater extent. We were also interested in examining possible differences between Junior-Juvenile (JUN) and Senior-Under ${ }^{23}$ (SEN) athletes in relation to the aforementioned responses. According to previous studies [2, 7, 8, 11, 12], we hypothesized a greater alteration in HR dynamics, a higher peak lactate and a slower lactate clearance following INT, despite our earlier result showing that both approaches share a significant final speed worsening, thus a positive pacing, with higher speed impairments for CON [1].

\section{MATERIAL AND METHODS}

\section{Participants}

Nineteen male highly-trained 800-m runners, belonging to either SEN ( $n=10)$ or JUN category $(n=9)$, took part in the study. The main characteristics of each group are presented in Table 1. No athlete was under medication during the investigation and all of them were previously advised with respect to resting hours, food intake and hydration. Vigorous exercise was not allowed for $48 \mathrm{~h}$ before the testing day and no training was permitted for $24 \mathrm{~h}$ before the testing day. The study was conformed to the Declaration of Helsinki. After the participants and their legal guardians were properly informed about the protocol, they gave their written consent to participate in the investigation, which was approved by the Research Ethics Committee of the University of Valencia.

\section{Experimental design}

Data were collected at the beginning of the competitive winter season, in two sessions, counterbalanced and randomized within 7 days of difference. A standardized warm-up was conducted to prepare participants for testing. On one session, athletes raced a race-pace continuous $600-\mathrm{m}$ run $(\mathrm{CON})$ at $100 \%$ of the equivalent speed for their previous season best performance in 800-m (SB); whereas in the other session athletes completed an interval training (INT) consisting of $2 \times 4 \times 200-\mathrm{m}$ at $102 \%$ of SB, with a 30 -s recovery between bouts and a 15 -min recovery between the sets. Total volume completed in both workouts was not matched, since the rationale for speed-endurance interval training in middle-distance athletics, in comparison to 
race-pace continuous workouts, is that total work completed must be greater, as the distance of the real event is split into much shorter distances interspersed with recovery periods $[13,16]$. Tests were conducted on a 400-m official outdoor track and within the same time slot to minimize external environmental influences. Temperature $\left(19.03 \pm 5.39{ }^{\circ} \mathrm{C}\right)$ and wind were registered $(0.56 \pm 1.63 \mathrm{~m} / \mathrm{s})$ thorough the test. Figure 1 presents a schematic view of the experimental protocol.

\section{Data acquisition}

On arrival to the track, athletes rested quietly for $10 \mathrm{~min}$ in a seated position to obtain baseline HR derived data; and immediately after each workout they were assisted to sit on the same chair, placed adjacent to the track, and remained still for the following $15 \mathrm{~min}$ to obtain post-exercise HR dynamics. HR measurements were performed using a Polar RS800 HR monitor together with a Polar Wearlink Wind electrode transmitter (Polar Electro, Kempele, Finland). This instrument had been previously validated for the accurate measurement of beat-to-beat (RR) interval in young and middle-aged men [17]. Seating position was chosen to avoid the risk of saturation of high frequency oscillations of HRV at rest [18], and respiratory rate was not controlled to not interfere with the athletes' ability or strategy to recover from the training. In either case, normal respiratory rate does not result in significantly different heart rate-derived indices compared with controlled breathing [19].

The procedures regarding performance assessment have already been described in detail [1]. Summarizing, running times and subsequent average speeds (AvSpeed) were registered with the aid of a CASIO HS EX-FH100 camera placed in the centre of the track, recording at $420 \mathrm{~Hz}$. An earlobe capillary blood sample was collected before the warm-up and 3,5 and 15 min following the workout $\left([\mathrm{La}]_{\text {pre, }},[\mathrm{La}]_{3},[\mathrm{La}]_{5},[\mathrm{La}]_{15}\right)$, using a Lactate Pro LT-1710 analyzer (Arkray Inc, Kyoto, Japan). Limits of agreement for Lactate Pro LT-1710 are -0.52 to $0.68 \mathrm{mmol} \mathrm{L}^{-1}$ [20]. The first two samples, 3 and 5 min after cessation, were chosen to detect the peak lactate concentration $\left([\mathrm{La}]_{\text {peak }}\right)[2,21]$, whereas the 15 min sample was collected to provide a value of the lactate clearance $\left([\mathrm{La}]_{\text {cle }}\right)[22]$. This latter was thus calculated as follows: $\left([\mathrm{La}]_{\text {peak }}-[\mathrm{La}]_{15}\right) /$ $[\mathrm{La}]_{\text {peak }} \times 100$. No other blood lactate measurements were made between the 5 th and the 15 th min of the recovery to minimize the possible disturbances in the beat-to-beat (RR) interval recording. Average $\mathrm{HR}\left(\mathrm{HR}_{\text {average }}\right)$, maximum $\mathrm{HR}\left(\mathrm{HR}_{\text {peak }}\right)$ and Rate of Perceived Exertion (RPE; scale 6-20) achieved during each workout were also considered. All participants were familiarized with RPE scoring prior to the study.

\section{HR Data treatment}

RR intervals were transferred to Polar Pro Trainer 5 software (Polar Electro, Kempele, Finland) and afterwards analyzed using Kubios HRV Analysis Software 2.0 (The Biomedical Signal and Medical Imaging Analysis Group, University of Kuopio, Finland). The whole analysis process was carried out by the same researcher to ensure consistency. Baseline data (PRE) were obtained from the last 5-min segment of the resting recording, whereas recovery data were extracted on four 180-s windows (Post1, Post2, Post3, Post4). These windows were the result of a balance between the requisite minimal number of RR intervals to ensure a correct analysis ( $\geq 250$ beats) [23] and the shortest possible duration. The initial $180 \mathrm{~s}$ were not evaluated to minimize the influence of pronounced downward trends [24]. Artefacts were identified and corrected using a 
medium level threshold. After estimating the trend of the RR interval series, artifact beats are identified as RR values deviating more than $0.25 \mathrm{~s}$ from this baseline and subsequently piecewise cubically interpolated, according to the manufacturer's recommendations [25]. Only recordings with $<3 \%$ of artefacts were considered. Recordings were also detrended (Smoothn priors, $\chi=$ 500) and resampled at $4 \mathrm{~Hz}$. The following indices were employed to analyze heart rate dynamics: mean HR; the root-mean-square difference of successive normal R-R intervals (RMSSD) to measure vagal reactivation; lnRMSSD to RR ratio, as a simultaneous measurement representative of vagal tone (RR) and modulation (lnRMSSD) [26]; nonlinear short-term scaling exponent (4-11 beats, $\mathrm{DFA}_{1}$ ) from Detrended Fluctuation Analysis to estimate the fractal-like correlation properties of the cardiac signal and assess sympathovagal balance [27]; and nonlinear Sample Entropy (SampEn) to provide an indication of the complexity of the timeseries under these circumstances [23]. Time domain indices were chosen instead of spectral indices because of their greater intraindividual reproducibility [28]; whereas SampEn and DFA have been stated as the most robust methods to assess complexity and fractal-like correlation properties of dynamic systems [14, 29].

\section{Statistical analyses}

Statistical analyses were carried out using the Statistical Package for the Social Sciences software (IBM SPSS Statistics for Windows, version 22.0, IBM Corp., Armonk, NY). After testing for normality (Shapiro-Wilk test), RMSSD was logarithmically transformed to allow parametric statistical comparisons (hereafter it will be referred as lnRMSSD). Firstly, the athletes' main characteristics (age, weight, height, personal best and best record in 800-m event during the previous season, and pre-exercise HR) were compared between JUN and SEN using an unpaired-samples Student's $t$-test. Afterwards, a three-factor repeated-measures ANOVA was conducted with one between factor, 'Age-category' (JUN vs SEN), and two within factors, 'Workout' (INT vs CON) and 'Time' (PRE, Post1, Post2, Post3, Post4), to assess the effect of the exercise on HR dynamics. The same procedure was employed to appraise differences in lactate concentration between resting condition and the $3^{\text {rd }}, 5^{\text {th }}$ and $15^{\text {th }}$ min of recovery. Additionally, differences in AvSpeed, RPE, $\mathrm{HR}_{\text {average}}, \mathrm{HR}_{\text {peak, }}[\mathrm{La}]_{\text {pre }},[\mathrm{La}]_{\text {peak }}$ and $[\mathrm{La}]_{\text {cle }}$ were evaluated through a two-factor repeated-measures ANOVA, with one between factor, 'Age-category' (JUN vs SEN), and one within factor, 'Workout' (INT vs CON). Whenever Mauchly's Sphericity test was violated, the necessary technical corrections were performed using the Greenhouse-Geisser test; and for each ANOVA, if a significant main effect or interaction was identified, Bonferroni post-hoc comparisons were conducted. The meaningfulness of the outcomes was estimated through the partial estimated effect size ( $\eta 2$ partial) for ANOVA and Hedges' $g$ effect size for pairwise comparisons. Small, medium and large effects were considered when $\eta 2$ partial was lower than 0.06 , between 0.06 and 0.14 , and greater than 0.14 , respectively. Threshold values for Hedges' $g$ were lower than 0.5 , between 0.5 and 0.8 , and greater than 0.8 . A $P$-value of $<0.05$ was considered statistically significant. Data are presented as means and standard deviations $( \pm \mathrm{SD})$.

\section{RESULTS}

All participants completed the experimental protocol; however, 3 athletes were excluded from the analysis due to an excessive number of artefacts $(>3 \%)$ in their post-exercise HR recordings. 
Table 1. Sample main characteristics (mean $\pm \mathrm{SD}$ )

\begin{tabular}{lrccc}
\hline & $n=19$ & Under23 - Senior SEN $=10$ & Juvenile - Junior JUN $=9$ & $P$ \\
\hline Age (years) & $21.36 \pm 5.26$ & $25.29 \pm 4.32$ & $17.00 \pm 0.66$ & $<0.001$ \\
Weight $(\mathrm{kg})$ & $67.18 \pm 4.27$ & $68.29 \pm 3.86$ & $65.94 \pm 4.58$ & 0.538 \\
Height $(\mathrm{cm})$ & $180.39 \pm 3.70$ & $180.40 \pm 4.19$ & $180.39 \pm 3.32$ & 0.832 \\
$P B(\mathrm{~s})$ & $116.55 \pm 5.53$ & $112.61 \pm 3.87$ & $120.92 \pm 3.33$ & $<0.001$ \\
$S B(\mathrm{~s})$ & $117.14 \pm 5.18$ & $113.75 \pm 4.08$ & $120.92 \pm 3.33$ & 0.002 \\
$\mathrm{HR}_{\text {preCON }}$ & $66.59 \pm 8.83$ & $63.83 \pm 10.53$ & $70.14 \pm 4.58$ & 0.129 \\
$\mathrm{HR}_{\text {preINT }}$ & $67.45 \pm 9.62$ & $64.20 \pm 11.12$ & $71.63 \pm 5.47$ & 0.164 \\
\hline
\end{tabular}

Abbreviations: $\mathrm{PB}$, personal best in 800 -m event; $\mathrm{SB}$, best record in $800-\mathrm{m}$ event during the previous season; $\mathrm{HR}_{\text {preCON }}$, average pre-exercise $\mathrm{HR}$ before the continuous workout; $\mathrm{HR}_{\text {preINT, }}$, average pre-exercise $\mathrm{HR}$ before the interval workout.

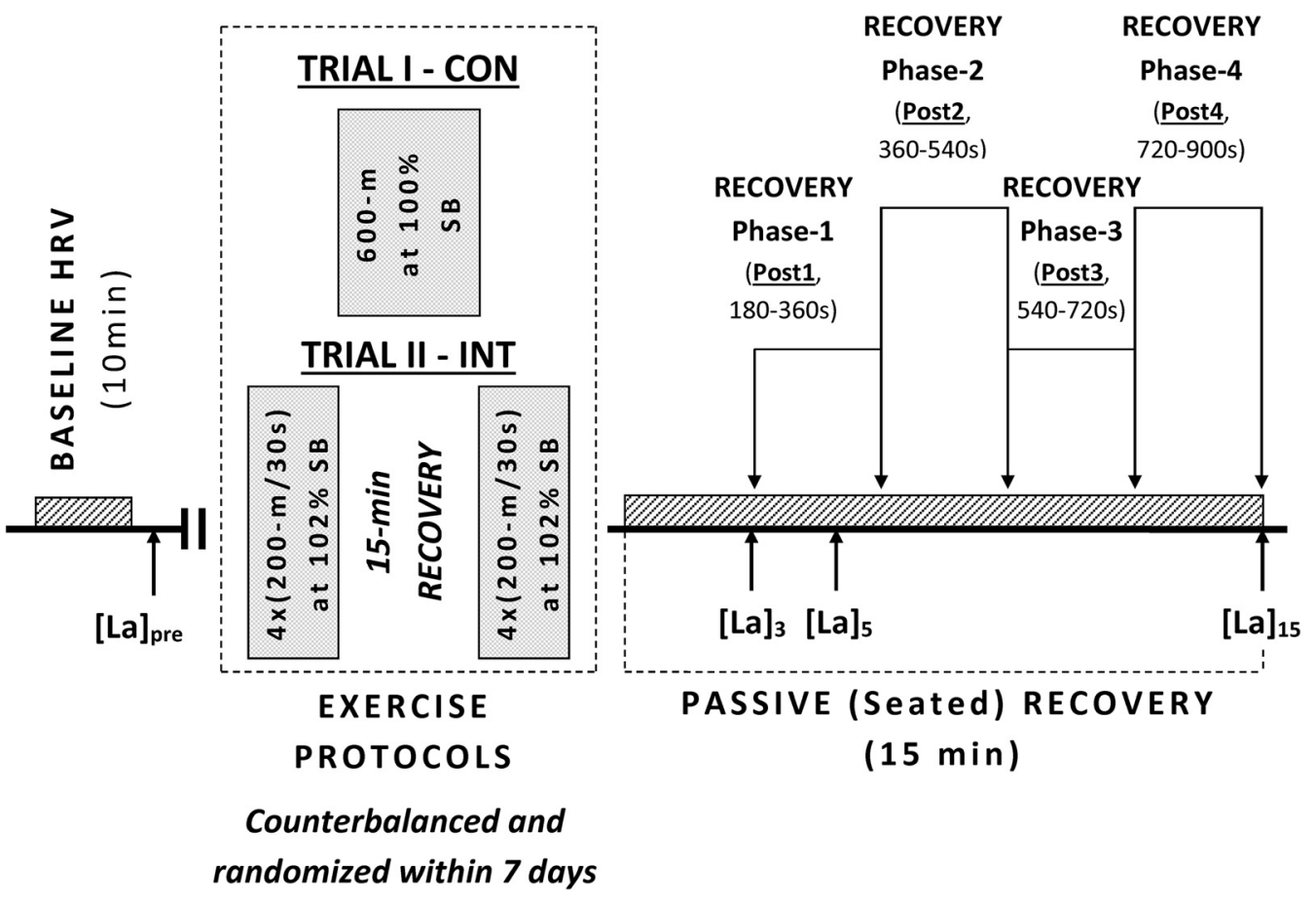

Fig. 1. Schematic view of the experimental protocol

The percentage of artifacts corrected in the remaining HR recordings were between 0 and $1 \%$. Exercise times in CON and INT workouts were $87.58 \pm 3.51 \mathrm{~s}$ and $236.29 \pm 10.23 \mathrm{~s}$, respectively. Values of AvSpeed, $\mathrm{HR}_{\text {peak, }} \mathrm{RPE},[\mathrm{La}]_{\text {pre }}$, $[\mathrm{La}]_{\text {peak }}$, $[\mathrm{La}]_{\text {cle }}$ following each workout are presented in Table 2. Following INT compared to CON, $[\mathrm{La}]_{\text {peak }}(P=0.001 ; g=1.14,95 \%$ CI: $0.40,1.91)$, and RPE $(P=0.001 ; g=1.15,95 \% \mathrm{CI}: 0.42,1.93)$ were higher; whereas AvSpeed was lower in 
Table 2. Descriptive data and values of lactate measures following CON and INT (mean \pm SD)

\begin{tabular}{|c|c|c|c|c|c|c|c|}
\hline & \multicolumn{2}{|c|}{ Race-pace continuous workout } & \multicolumn{2}{|c|}{ Interval workout } & \multirow[b]{2}{*}{$\begin{array}{l}\text { Workout } \\
\text { factor }\end{array}$} & \multirow[b]{2}{*}{$\begin{array}{l}\text { Age-category } \\
\text { factor }\end{array}$} & \multirow{2}{*}{$\begin{array}{c}\text { Workout } \times \text { Age } \\
\text { Category } \\
\text { interaction }\end{array}$} \\
\hline & $\begin{array}{c}\text { Juvenile - } \\
\text { Junior }(n=7)\end{array}$ & $\begin{array}{c}\text { Under23- } \\
\text { Senior }(n=9)\end{array}$ & $\begin{array}{c}\text { Juvenile - } \\
\text { Junior }(n=7)\end{array}$ & $\begin{array}{c}\text { Under23- } \\
\text { Senior }(n=9)\end{array}$ & & & \\
\hline AvSpeed $\left(\mathrm{m} \mathrm{s}^{-1}\right)$ & $6.72 \pm 0.18$ & $6.97 \pm 0.31$ & $6.60 \pm 0.18$ & $6.92 \pm 0.32$ & $P=0.058$ & $\begin{array}{c}P=0.041 \\
\text { SEN }>\text { JUN }\end{array}$ & $P=0.460$ \\
\hline $\mathbf{H R}_{\text {average }}(\mathrm{bpm})$ & $183.01 \pm 7.68$ & $173.22 \pm 9.91$ & $185.57 \pm 4.82$ & $177.89 \pm 8.64$ & $P=0.145$ & $P=0.265$ & $P=0.439$ \\
\hline $\mathbf{H R}_{\text {peak }}(\mathrm{bpm})$ & $191.14 \pm 5.43$ & $178.55 \pm 11.59$ & $187.86 \pm 5.11$ & $181.78 \pm 7.95$ & $P=0.115$ & $P=0.385$ & $P=0.439$ \\
\hline RPE & $15.43 \pm 1.51$ & $15.67 \pm 2.40$ & $17.29 \pm 1.11$ & $18.01 \pm 1.87$ & $\begin{array}{l}P=0.001 \\
\text { INT }>\text { CON }\end{array}$ & $P=0.575$ & $P=0.580$ \\
\hline$[\mathrm{La}]_{\text {pre }}\left(\mathrm{mmol} \mathrm{L}^{-1}\right)$ & $1.39 \pm 0.31$ & $1.42 \pm 0.34$ & $1.34 \pm 0.31$ & $1.34 \pm 0.27$ & $P=0.570$ & $P=0.872$ & $P=0.869$ \\
\hline$[\mathrm{La}]_{\text {peak }}\left(\mathrm{mmol} \mathrm{L}^{-1}\right)$ & $13.27 \pm 1.24$ & 14. $27 \pm 2.06$ & $15.69 \pm 1.06$ & $15.38 \pm 0.98$ & $\begin{array}{l}P=0.001 \\
\text { INT }>\text { CON }\end{array}$ & $P=0.592$ & $P=0.094$ \\
\hline$[\mathrm{La}]_{\mathrm{cle}}(\%)$ & $2.70 \pm 3.66$ & $1.23 \pm 2.53$ & $5.10 \pm 6.17$ & $2.02 \pm 2.38$ & $P=0.328$ & $P=\mathbf{0 . 0 8 7}$ & $P=0.616$ \\
\hline
\end{tabular}

Abbreviations: AvSpeed, average speed during the test; $\mathrm{HR}_{\text {average, }}$ average $\mathrm{HR}$ achieved during the test; $\mathrm{HR}_{\text {peak }}$, maximum HR achieved during the test; RPE, Rate of Perceived Exertion; $[\mathrm{La}]_{\text {pre, }}$ lactate accumulation at baseline; $[\mathrm{La}]_{\text {peak, }}$ maximum lactate accumulation during the recovery period; $[\mathrm{La}]_{\text {cle, }}$ percentage difference between lactate at the $15^{\text {th }}$ minute of recovery and $[\mathrm{La}]_{\text {peak }}$; SEN, Senior-Under23 athletes; JUN, Junior-Juvenile athletes; CON, Race-Pace Continuous workout; INT, Interval workout. 
JUN compared to SEN $(P=0.041 ; g=1.08,95 \%$ CI: $0.35,1.85)$. No 'Workout x Age-category' interactions were noted.

Figure 2 illustrates the time course of HR dynamics indices during recovery from INT and CON workouts. Univariate contrast analysis showed a significant effect for 'Time' on HR $[\mathrm{F}=211.96 ; P=0.001 ; \eta 2$ partial $=0.94,95 \% \mathrm{CI}: 0.86,0.96] ; \operatorname{lnRMSSD}[\mathrm{F}=68.82 ; P=0.001$; $\eta 2$ partial $=0.83,95 \%$ CI: $0.66,0.88], \operatorname{lnRMSSD} / \mathrm{RR}[\mathrm{F}=11.06 ; P=0.001 ; \eta 2$ partial $=0.44$, 95\% CI: $0.21,0.56]$, and $\mathrm{DFA}_{1}[\mathrm{~F}=6.63 ; P=0.002 ; \eta 2$ partial $=0.32,95 \% \mathrm{CI}: 0.06$, 0.49]; whereas 'Workout' condition significantly affected lnRMSSD [F $=5.11 ; P<0.040$; $\eta 2$ partial $=0.26,95 \% \mathrm{CI}: 0.01,0.54]$, and $\mathrm{DFA}_{1}[\mathrm{~F}=8.73 ; P=0.010 ; \eta 2$ partial $=0.38,95 \%$ CI: 0.03, 0.62]. No significant 'Age-category' effects were revealed for HR dynamics indices, although SampEn was significantly affected by 'Time $\times$ Age-category' $[\mathrm{F}=3.10 ; P=0.046$; $\eta 2$ partial $=0.18,95 \% \mathrm{CI}: 0.01,0.36]$ and 'Time $\times$ Workout' conditions $[\mathrm{F}=2.53 ; P=0.049$; $\eta 2$ partial $=0.15,95 \% \mathrm{CI}: 0.01,0.28]$. There were no other significant interaction effects. Bonferroni adjusted pairwise comparisons showed that HR significantly dropped from Post1 to Post2 following both INT $(P=0.001 ; g=1.20,95 \% \mathrm{CI}: 0.46,1.98)$ and CON $(P=0.001$; $g=0.90,95 \%$ CI: $0.19,1.65)$ and then remained constant without significant changes from
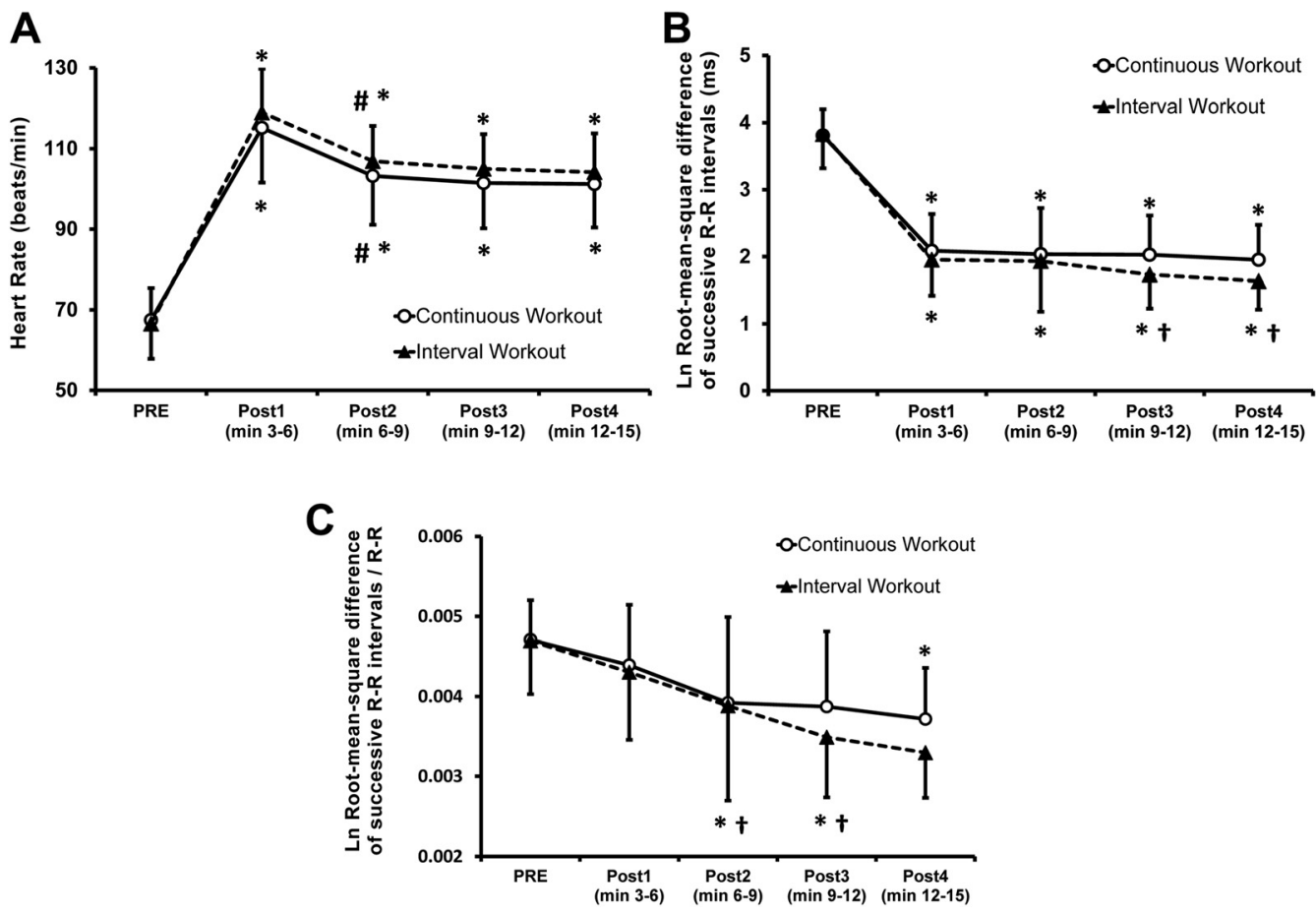

Fig. 2. HR (panel A), LnrMSSD (panel B) and LnrMSSD/RR (panel C) behavior during recovery from INT and CON.

Open circles represent data from CON and solid triangles represent data from INT.

* Significantly different from baseline condition $(P<0.05)$; ${ }^{*}$ Significantly different from the preceding recovery phase $(P<0.05) ;{ }^{\dagger}$ Significantly different from CON $(P<0.05)$ 

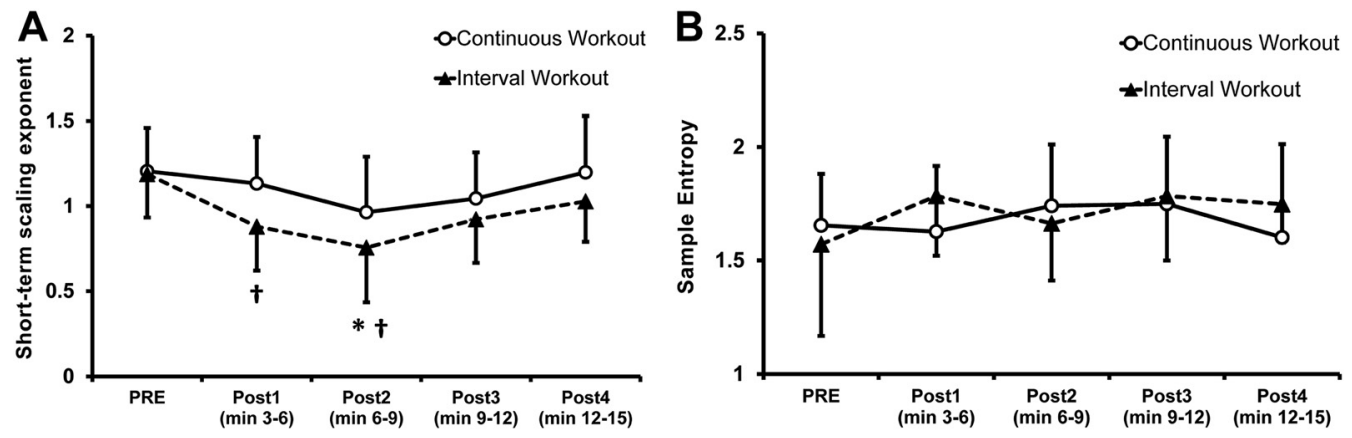

Fig. 3. $\mathrm{DFA}_{1}$ (panel A) and Sample Entropy (panel B) behavior during recovery from INT and CON. Open circles represent data from CON and solid triangles represent data from INT.

* Significantly different from baseline condition $(P<0.05)$; ${ }^{*}$ Significantly different from the preceding recovery phase $(P<0.05){ }^{\dagger}$ Significantly different from $\operatorname{CON}(P<0.05)$

Post2 to Post4 (Fig. 2A). Besides, their values were significantly greater during the whole recovery compared to baseline condition $(P=0.001)$. Similarly, lnRMSSD kept being significantly lower during the 15 -min recovery $(P=0.001)$; although lnRMSDD, unlike HR, did not change from Post1 to Post2 (Fig. 2B). LnRMSSD/RR was significantly different from baseline only at Post 3 (following INT; $P=0.005$ ) and Post 4 (following both workouts; CON: $P=0.005$, INT: $P=0.001$ ) (Fig. 2C). No significant changes were noted between adjacent recovery periods. $\mathrm{DFA}_{1}$ showed a non-significant trend of decreasing values below the baseline level during Post1 and Post2 (the difference with PRE reached statistical significance, $P=0.010$ only at Post 2 following INT) and increased also non-significantly from this point onwards (Fig. 3A). Conversely, no differences between resting and post-exercise conditions were noted for SampEn (Fig. 3B). Moreover, lnRMSSD displayed lower values following INT compared to CON at Post3

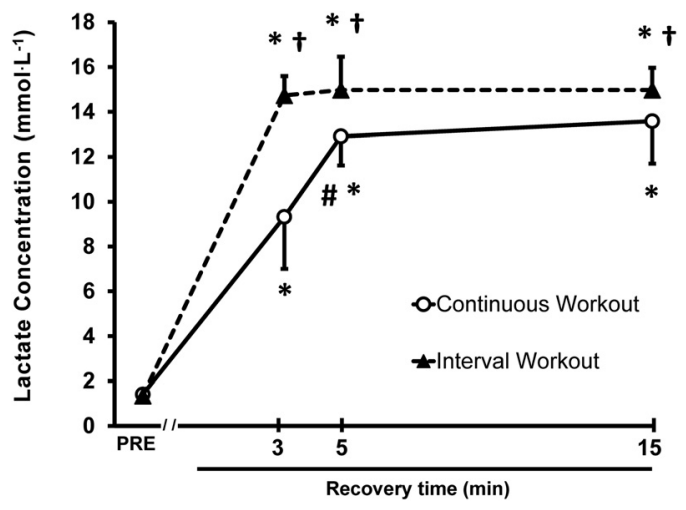

Fig. 4. Lactate behavior during recovery from INT and CON.

Open circles represent data from CON and solid triangles represent data from INT.

* Significantly different from baseline condition $(P<0.05)$; ${ }^{*}$ Significantly different from the preceding recovery phase $(P<0.05) ;{ }^{\dagger}$ Significantly different from CON $(P<0.05)$ 
$(P=0.002 ; g=0.53,95 \%$ CI: $0.17,1.25)$ and Post $4(P=0.016 ; g=0.66,95 \%$ CI: $0.04,1.38)$. LnRMSSD/RR also showed lower values following INT compared to CON at Post3 $(P=0.013$; $g=0.43,95 \%$ CI: $1.14,0.25)$ and Post $4(P=0.032 ; g=0.68,95 \%$ CI: 1.40, 0.03). Lastly, DFA 1 was lesser following INT compared to CON at Post1 $(P=0.004 ; g=0.91,95 \%$ CI: $0.20,1.67)$ and Post2 $(P=0.036 ; g=0.60,95 \%$ CI: $0.10,1.32)$.

Figure 4 displays the time course of lactate concentration during recovery from INT and CON workouts. [La] remained significantly higher compared to baseline condition during the entire recovery following both INT $(P=0.001)$ and $\operatorname{CON}(P=0.001)$, although values after INT workout were significantly greater than CON ones at all recovery time points: $[\mathrm{La}]_{3}(P=$ $0.001 ; g=3.01,95 \%$ CI: $2.03,4.11) ;[\mathrm{La}]_{5}(P=0.001 ; g=1.45,95 \%$ CI: $0.69,2.26) ;[\mathrm{La}]_{15}(P=$ $0.004 ; g=0.91,95 \% \mathrm{CI}: 0.19,1.66)$. Moreover, a significant increase from $[\mathrm{La}]_{3}$ to $[\mathrm{La}]_{5}$ was noted following CON $(P<0.001 ; g=1.85,95 \%$ CI: $1.05,2.74)$ but not after INT workout.

\section{DISCUSSION}

In a previous paper we delved into the advantages and disadvantages of race-pace continuous vs. interval workouts regarding performance and pacing improvement in 800-m athletics [1]; meanwhile, the main aim of the present study was to assess HR dynamics during the immediate 15-min recovery together with peak lactate and 15-min post-exercise lactate clearance, and to examine possible differences between JUN and SEN athletes in relation to the aforementioned responses. As we hypothesized, a greater alteration in linear (i.e., rMSSD) and nonlinear (i.e., DFA $_{1}$ ) HR dynamics was observed following INT compared to CON. Besides, INT provoked a higher post-exercise $[\mathrm{La}]_{\text {peak }}$ than $\mathrm{CON}$, thus confirming our second hypothesis. However, contrary to our expectations, no differences were found in $[\mathrm{La}]_{c l e}$ between workouts. Lastly, even for highly trained athletes, $15 \mathrm{~min}$ seem insufficient to recover vagally mediated HRV (i.e., lnRMSSD) following a supramaximal (i.e., performed at $>100 \%$ of $\mathrm{v}_{\mathrm{V}} \mathrm{O}_{2 \max }$ ) running workout, either continuous or intervallic. Notwithstanding, given that we failed to obtain actual $\mathrm{vVO}_{2} \mathrm{max}$ data of the participants, further studies are required to confirm this assumption.

Current results agree with other previous studies displaying a larger reduction in vagal modulation following an interval workout in comparison with a continuous one $[7,8,11,12]$, and add to previous investigations the fact that fractal scaling properties also appear to be greatly disturbed after an intervallic exercise. Only Kaikkonen et al. [10] have shown a greatly impaired HRV after a continuous exercise; however, in competitive athletic training, it is rather unusual to perform an intermittent and a continuous workout at the same intensity (in relation to $\mathrm{vVO}_{2 \max }$ ) for the same total time, as these authors employed for their study. Interval approaches, compared to race-pace continuous workouts, usually split the distance of the real event into much shorter distances interspersed with recovery periods; and so this layout enables a greater total volume for this kind of training. Neither are our results in accordance with Cyprian \& Vala [9], who found no differences in HRV recovery following a continuous graded maximal test compared to an intermittent all-out exercise. Several reasons could explain this disagreement: participants in their study were not engaged in any competitive sport [6], workouts were performed on a bicycle ergometer [30] and HRV was quantified using spectral analysis [28]. Alongside, we found no difference in $\mathrm{HR}_{\text {average }}$ and $\mathrm{HR}_{\text {peak }}$ during INT compared to CON. Hence, the larger reduction in vagal modulation observed following INT does not seem to be 
attributable to a greater sympathetic involvement during the workout, as previously suggested $[8,11,12]$. Notwithstanding, because of the abovementioned inherent characteristics of the two workouts employed, total distance covered in INT was higher (1,600 m vs $600 \mathrm{~m})$, so workload was greater for the intervallic protocol and thus the magnitude of neurocardiac control alteration could be expected to be greater [31]. However, in a recent study by Kliszczewicz et al. [32] no differences were found in post-exercise HRV between a short $(<5 \mathrm{~min})$ and a long (15 $\mathrm{min})$ high-intensity functional training among recreational athletes. Further investigation is needed to clarify how the dose of supramaximal exercise affects cardiac autonomic recovery.

On the other hand, our results match the depressed vagal reactivation previously reported among inactive individuals or moderately trained athletes after a high-intensity exercise [7-9, 33]. However, they are in disagreement with a recent review [6], where authors found that postexercise suppression of vagal modulation is reduced to small or trivial after $15 \mathrm{~min}$ in highly trained athletes. Interestingly, we already showed a similar blunted vagal response following a sport-specific supramaximal test among highly trained judokas [16]. Alongside, $\mathrm{DFA}_{1}$ displayed a shift towards a more random HR dynamics behavior (i.e., $\mathrm{DFA}_{1}$ closer to 0.5 ) during the first 9 min of recovery (i.e. Post1 and Post2) and a progressive restoration towards baseline values from this point onwards. This more random behavior during the initial phase of recovery could be due to a concomitant activation or an accentuated sympathovagal outflow [34]. Interestingly, this pattern is completely opposite to that found in previous studies assessing recovery from high-intensity workouts $[16,31]$, where $\mathrm{DFA}_{1}$ showed a more predictable $\mathrm{HR}$ signal (i.e., $\mathrm{DFA}_{1}$ closer to 1.5) during post-exercise condition compared to baseline. In addition, SampEn showed no change from pre-exercise to post-exercise condition, coinciding with a recent study where HR dynamics were assessed following a supramaximal Judo test [16]. Unlike those results and ones from current research, previous investigations involving non-athletes did show a persistent reduction in SampEn 5-10 min after either a supramaximal effort or a resistance training session $[35,36]$. Even more, in Millar et al.'s study [31], SampEn remained altered for $2 \mathrm{~h}$ after a single Wingate test; and in Hoshi et al.'s study [37], Shannon Entropy was not restored until 80 min following a constant velocity exhaustive test. Overall, although entropy and fractal measures of HR signal are modulated by vagal and sympathetic outflows [27], our results reinforce the notion that they reflect more stable properties of HR dynamics [14]; and therefore, their postexercise recovery timeline follow a different pattern in comparison to vagal reactivation and as a function of the athletes' level of performance.

$[\mathrm{La}]_{\text {peak }}$ values obtained following both INT and CON fell within the formerly reported range after high-intensity workouts usually employed in 400-m training [2]. They were also similar to those observed following a time trial $800-\mathrm{m}$ race [13]. Nevertheless, INT provoked a higher post-exercise $[\mathrm{La}]_{\text {peak }}$ than $\mathrm{CON}$. This in agreement with previous studies $[2,7,8,21]$. However, despite this greater $[\mathrm{La}]_{\text {peak }}$ following INT, no differences were found in $[\mathrm{La}]_{\text {cle }}$ between workouts. Actually, $[\mathrm{La}]_{\text {cle }}$ was almost nonexistent during the 15 -min recovery (between 0 and $16 \%)$ and no significant differences appeared between recovery time points $\left([\mathrm{La}]_{3},[\mathrm{La}]_{5}\right.$, $[\mathrm{La}]_{15}$ ) following either INT or CON. The fact that athletes remained seated during the whole recovery period in order to match baseline conditions regarding body position probably prevented them from achieving a larger lactate removal $[2,21,38]$. This scenario, similar to that depicted by Bastos et al. [22] and ourselves in a former study [16] coincides with the absence of changes in InRMSSD during the recovery period and lead us to suggest that post-exercise lactate is closely interrelated to both vagal reactivation and fractal scaling properties restoration, as 
previously postulated $[16,33,37]$. Meanwhile, complexity properties of HR dynamics (i.e., SampEn) seem not to keep this interrelation with post-exercise lactate.

These results should, however, be considered with regard to some methodological limitations. The high specificity of the intervention, as well as the limited availability of $800-\mathrm{m}$ elite athletes of different age-categories with a similar periodization and competition period made the experimental procedure quite challenging and prevented us from amassing a larger sample. Secondly, we assume that a longer recovery period may have allowed us to observe lactate clearance differences between workouts. In addition, we acknowledge that the two workouts we compared were not equal regarding volume, intensity and work-to-rest ratio due to their inherent characteristics, thus limiting the interval validity of the study. On the other hand, we could not measure respiratory rate and tidal volume during HR recordings, therefore we are unaware of how respiratory arrhythmia could have affected HRV measures (especially rMSSD). Lastly, although race-pace workouts were supposed to be performed at an intensity equal or even greater to the velocity corresponding to $\dot{\mathrm{V}}_{2}$ max, this assumption could not be experimentally checked.

\section{CONCLUSIONS}

Regarding the suitability of the two workouts analyzed, race-pace continuous workouts, when performed on a basis of a single run conducted at competitive pace and covering between 50 and $75 \%$ of the real competition distance, provoke a lesser alteration in HR dynamics and a lower post-exercise peak lactate compared to speed-endurance interval workouts. Therefore, it seems advisable to perform a second repetition in race-pace continuous workouts usually employed in middle-distance athletics training to induce a greater training effect. In addition, irrespectively of HR dynamics and lactate responses, there are other variables, such as pacing-adjustment learning, for which race-pace continuous workouts may be preferable against intervallic approaches.

Moreover, our results evince that vagally mediated HRV remains significantly reduced $15 \mathrm{~min}$ following the two workouts analyzed, even when highly trained athletes are involved. Similarly, lactate clearance is minimal (between 0 and 16\%) during these $15 \mathrm{~min}$ when a passive recovery is adopted. As a consequence, neither a restoration of HRV nor a lactate clearance could be assumed when such a recovery is applied between sets of repetitions in middle-distance athletics workouts.

\section{ACKNOWLEDGMENTS}

The authors are grateful to all the athletes, coaches and collaborators who participated in this study.

\section{REFERENCES}

1. Blasco-Lafarga C, Montoya-Vieco A, Martinez-Navarro I, Mateo-March M, Gallach JE. Six hundred meterrun and broken 800 's contribution to pacing improvement in eight hundred meter-athletics: role of expertise and training implications. J Strength Cond Res 2013 Sep; 27(9): 2405-13. 
2. Saraslanidis PJ, Manetzis CG, Tsalis GA, Zafeiridis AS, Mougios VG, Kellis SE. Biochemical evaluation of running workouts used in training for the 400-m sprint. J Strength Cond Res 2009 Nov; 23(8): $2266-71$.

3. Gajer B. Le 800 mètres: analyse descriptive et entraînement. Paris: Institut national du sport et de l'éducation physique; 2000.

4. García-Verdugo M, Landa L. Atletismo 4. La preparación del corredor de resistencia. Madrid: Real Federación Española de Atletismo; 2005.

5. Laursen PB. Training for intense exercise performance: high-intensity or high-volume training? Scand J Med Sci Sports 2010 Oct; 20(Suppl. 2): 1-10.

6. Stanley J, Peake JM, Buchheit M. Cardiac parasympathetic reactivation following exercise: implications for training prescription. Sports Med 2013 Dec; 43(12): 1259-77.

7. Buchheit M, Al Haddad H, Millet GP, Lepretre PM, Newton M, Ahmaidi S. Cardiorespiratory and cardiac autonomic responses to 30-15 intermittent fitness test in team sport players. J Strength Cond Res 2009 Jan; 23(1): 93-100.

8. Buchheit M, Laursen PB, Ahmaidi S. Parasympathetic reactivation after repeated sprint exercise. Am J Physiol Heart Circ Physiol 2007 Jul; 293(1): H133-41.

9. Cipryan L, Vala R. Cardiac autonomic regulation after continuous and intermittent maximal exercise interventions. J Sports Med Phys Fitness 2015 May; 55(5): 495-505.

10. Kaikkonen P, Rusko H, Martinmaki K. Post-exercise heart rate variability of endurance athletes after different high-intensity exercise interventions. Scand J Med Sci Sports 2008 Aug; 18(4): 511-9.

11. Mourot L, Bouhaddi M, Tordi N, Rouillon JD, Regnard J. Short- and long-term effects of a single bout of exercise on heart rate variability: comparison between constant and interval training exercises. Eur J Appl Physiol 2004 Aug; 92(4-5): 508-17.

12. Niewiadomski W, Gasiorowska A, Krauss B, Mroz A, Cybulski G. Suppression of heart rate variability after supramaximal exertion. Clin Physiol Funct Imaging 2007 Sep; 27(5): 309-19.

13. Billat V, Hamard L, Koralsztein JP, Morton RH. Differential modeling of anaerobic and aerobic metabolism in the 800-m and 1,500-m run. J Appl Physiol (1985) 2009 Aug; 107(2): 478-87.

14. Nicolini P, Ciulla MM, De Asmundis C, Magrini F, Brugada P. The prognostic value of heart rate variability in the elderly, changing the perspective: from sympathovagal balance to chaos theory. Pacing Clin Electrophysiol 2012 May; 35(5): 622-38.

15. Millar PJ, Rakobowchuk M, Adams MM, Hicks AL, McCartney N, MacDonald MJ. Effects of short-term training on heart rate dynamics in individuals with spinal cord injury. Auton Neurosci 2009 Oct 5; 150(1-2): $116-21$.

16. Blasco-Lafarga C, Martinez-Navarro I, Mateo-March M. Is baseline cardiac autonomic modulation related to performance and physiological responses following a supramaximal Judo test? PLoS One 2013; 8(10): e78584.

17. Wallen MB, Hasson D, Theorell T, Canlon B, Osika W. Possibilities and limitations of the Polar RS800 in measuring heart rate variability at rest. Eur J Appl Physiol 2012 Mar; 112(3): 1153-65.

18. Kiviniemi AM, Hautala AJ, Seppanen T, Makikallio TH, Huikuri HV, Tulppo MP. Saturation of high-frequency oscillations of R-R intervals in healthy subjects and patients after acute myocardial infarction during ambulatory conditions. Am J Physiol Heart Circ Physiol 2004 Nov; 287(5): H1921-7.

19. Bloomfield DM, Magnano A, Bigger JT, Jr., Rivadeneira H, Parides M, Steinman RC. Comparison of spontaneous vs. metronome-guided breathing on assessment of vagal modulation using RR variability. Am J Physiol Heart Circ Physiol 2001 Mar; 280(3): H1145-50.

20. Pyne DB, Boston T, Martin DT, Logan A. Evaluation of the lactate pro blood lactate analyser. Eur J Appl Physiol 2000 May; 82(1-2): 112-6. 
21. Peake JM, Tan SJ, Markworth JF, Broadbent JA, Skinner TL, Cameron-Smith D. Metabolic and hormonal responses to isoenergetic high-intensity interval exercise and continuous moderate-intensity exercise. Am J Physiol Endocrinol Metab 2014 Oct 1; 307(7): E539-52.

22. Bastos FN, Vanderlei LC, Nakamura FY, Bertollo M, Godoy MF, Hoshi RA, et al. Effects of cold water immersion and active recovery on post-exercise heart rate variability. Int J Sports Med 2012 Nov; 33(11): 873-9.

23. Richman JS, Moorman JR. Physiological time-series analysis using approximate entropy and sample entropy. Am J Physiol Heart Circ Physiol 2000 Jun; 278(6): H2039-49.

24. Task Force. Heart rate variability. Standards of measurement, physiological interpretation, and clinical use. Task force of the European Society of Cardiology and the North American Society of pacing and electrophysiology. Eur Heart J 1996 Mar; 17(3): 354-81.

25. Tarvainen M, Niskanen J, Lipponen J, Ranta-aho P, Karjalainen P, editors. Kubios HRV-a software for advanced heart rate variability analysis. 4th European Conference of the International Federation for Medical and Biological Engineering; 2008; Antwerp, Belgium: Springer.

26. Plews DJ, Laursen PB, Stanley J, Kilding AE, Buchheit M. Training adaptation and heart rate variability in elite endurance athletes: opening the door to effective monitoring. Sports Med 2013 Sep; 43(9): 773-81.

27. Tulppo MP, Kiviniemi AM, Hautala AJ, Kallio M, Seppanen T, Makikallio TH, et al. Physiological background of the loss of fractal heart rate dynamics. Circulation 2005 Jul 19; 112(3): 314-9.

28. Al Haddad H, Laursen PB, Chollet D, Ahmaidi S, Buchheit M. Reliability of resting and postexercise heart rate measures. Int J Sports Med 2011 Aug; 32(8): 598-605.

29. PerkiöMäKi JS, Mäkikallio TH, Huikuri HV. Fractal and complexity measures of heart rate variability. Clin Exp Hypertens 2005; 27(2-3): 149-58.

30. Esco MR, Flatt AA, Williford HN. Postexercise heart rate variability following treadmill and cycle exercise: a comparison study. Clin Physiol Funct Imaging 2015 Oct 7.

31. Millar PJ, Rakobowchuk M, McCartney N, MacDonald MJ. Heart rate variability and nonlinear analysis of heart rate dynamics following single and multiple Wingate bouts. Appl Physiol Nutr Metab 2009 Oct; 34(5): 875-83.

32. Kliszczewicz B, Williamson C, Bechke E, McKenzie M, Hoffstetter W. Autonomic response to a short and long bout of high-intensity functional training. J Sports Sci 2018 Aug; 36(16): 1872-9.

33. Kaikkonen P, Hynynen E, Mann T, Rusko H, Nummela A. Heart rate variability is related to training load variables in interval running exercises. Eur J Appl Physiol 2012 Mar; 112(3): 829-38.

34. Tulppo MP, Kiviniemi AM, Hautala AJ, Kallio M, Seppanen T, Tiinanen S, et al. Sympatho-vagal interaction in the recovery phase of exercise. Clin Physiol Funct Imaging 2011 Jul; 31(4): 272-81.

35. Goulopoulou S, Fernhall B, Kanaley JA. Hemodynamic responses and linear and non-linear dynamics of cardiovascular autonomic regulation following supramaximal exercise. Eur J Appl Physiol 2009 Mar; 105(4): 525-31.

36. Heffernan KS, Sosnoff JJ, Jae SY, Gates GJ, Fernhall B. Acute resistance exercise reduces heart rate complexity and increases QTc interval. Int J Sports Med 2008 Apr; 29(4): 289-93.

37. Hoshi RA, Vanderlei LCM, de Godoy MF, Bastos FDN, Netto J, Jr., Pastre CM. Temporal sequence of recovery-related events following maximal exercise assessed by heart rate variability and blood lactate concentration. Clin Physiol Funct Imaging 2017 Sep; 37(5): 536-43.

38. Miladi I, Temfemo A, Mandengue SH, Ahmaidi S. Effect of recovery mode on exercise time to exhaustion, cardiorespiratory responses, and blood lactate after prior, intermittent supramaximal exercise. J Strength Cond Res 2011 Jan; 25(1): 205-10. 